九州大学学術情報リポジトリ

Kyushu University Institutional Repository

Fireworks Algorithm for Multimodal Optimization Using a Distance-based Exclusive Strategy

YU, Jun

Graduate School of Design, Kyushu University

Takagi, Hideyuki

Faculty of Design, Kyushu University, Kyushu University

TAN, Ying

School of Electronics Engineering and Computer Science, Peking University

ht tp://hdl. hand le. net/2324/2230996

出版情報 : 2019-06-10

バージョン：

権利関係 : 


\section{Fireworks Algorithm for Multimodal Optimization Using a Distance-based Exclusive Strategy}

\author{
Jun YU \\ Graduate School of Design \\ Kyushu University \\ Fukuoka, Japan \\ JSPS Research Fellow \\ yujun@kyudai.jp
}

\author{
Hideyuki TAKAGI \\ Faculty of Design \\ Kyushu University \\ Fukuoka, Japan \\ URL: http://www.design.kyushu-u.ac.jp/ takagi/
}

\author{
Ying TAN \\ School of Electronics Engineering \\ and Computer Science, Peking University \\ Beijing, China \\ ytan@pku.edu.cn
}

\begin{abstract}
We propose a distance-based exclusive strategy to extend fireworks algorithm as a niche method to find out multiple global/local optima. This strategy forms sub-groups consisting of a firework individual and its generated spark individuals, each sub-group is guaranteed not to search overlapped areas each other. Finally, firework individuals are expected to find different global/local optima. The proposed strategy checks the distances between a firework and other fireworks which fitness is better than that of the firework. If the distance between two firework individuals is shorter than the sum of their searching radius, i.e. amplitude of firework explosions, these two firework individuals are considered to search overlapped area. Thus, the poor firework is removed and replaced by its opposite point to track multiple optima. To evaluate the performance of our proposed strategy, enhanced fireworks algorithm (EFWA) is used as a baseline algorithm and combined with our proposal. We design a controlled experiment, and run EFWA and (EFWA + our proposal) on 8 benchmark functions from CEC 2015 test suite, that is dedicated to single objective multi-niche optimization. The experimental results confirmed that the proposed strategy can find multiple different optima in one trial run.
\end{abstract}

Index Terms-fireworks algorithm, multimodal optimization, niching, exclusive strategy

\section{INTRODUCTION}

Evolutionary computation (EC) is a form of populationbased optimization techniques and simulates biological evolution and the survival of the fittest repeatedly to find the global optimum. Many practitioners gained novel inspirations from natural phenomenon and human society to propose efficient EC algorithms, such as differential evolution (DE) [1], particle swarm optimization (PSO) [2], fireworks algorithm (FWA) [3] and others [4], [5], [6]. These EC algorithms have attracted extensive attentions thanks to their various excellent features, such as, simplicity, parallelism and intelligence. Realistic optimization problems become so complicate and difficult to model mathematically that traditional methods, e.g. linear programming and Newton method, are powerless to solve them. On the other hand, EC algorithms have been applied to these realistic problems widely because of their various powerful characteristics. For example, the nose cone of the N700 series Shinkansen is redesigned by genetic algorithm to reduce pressure wave caused by a tunnel energy consumption.
Most of realistic optimization problems are multimodal, and sometimes their many different optima or sub-optima are necessary to be find out rather than only one optimum. Unfortunately, enough attention has not paid to this kind of niche in the EC community, and most practitioners focus on only finding the global optimum and ignore other acceptable candidate solutions. Actually, some users not only want to get the global optimum but also expect to have several available sub-optima for making a final decision. Niching techniques as a highly focused option are the extension of EC and can find multiple optima during one trial run.

So far, there are many well-known niching methods that have been proposed in the past few decades, including clearing [7], restricted tournament selection [8], crowding [9], fitness sharing [10], species [11] and others [12]. Although these methods have shown satisfactory performance, they need introduce new parameters to track multiple optima. It increases the complexity and computational cost, and there are still some limitations that need to be addressed, e.g. low convergence, high computational cost and parameter tuning.

The main objective of this paper is to extend original FWA to slove multimodal optimization problems by introducing a distance-based exclusive strategy to find out multiple different optima. The proposed strategy uses the characteristics of FWA to avoid fireworks searching in the same area and motivate them to converge toward different optima. Finally, each firework can maintain a niching at the end of one trial run. Subsequently, we analyze the performance and applicability of our proposed strategy. We also introduce some topics which are open to discussion.

Following this introductory section, we summarize the implementation mechanism of the original FWA in the Section II. The proposed strategy is presented in detail in the Section III. We compare our proposal with the famous fitness sharing method using 8 benchmark functions from CEC 2015 test suite in the Section IV. Finally, we discuss and analyze the effects of our proposal and conclude our works in the Sections V and VI, respectively. 


\section{Fireworks Algorithm}

When a firework is launched into the sky, it generates many sparks around itself to form a beautiful pattern. Inspired by the explosion of real fireworks, the explosion process can be abstracted as a local search around a particular point (firework). In summary, FWA generates some initial firework individuals randomly and adaptively determines a search range or explosion amplitude as well as the number of generated spark individuals for each firework individual according to its fitness. Then, each firework generates a certain amount of spark individuals within its explosion amplitude as an explosion operation. Finally, all individuals including spark individuals and current firework individuals are mixed together to select the next generation. FWA simulates this explosion process iteratively until a termination condition is satisfied. Fig. 1 illustrates the process of conventional FWA that consists principally of three operations: explosion, mutation and selection.

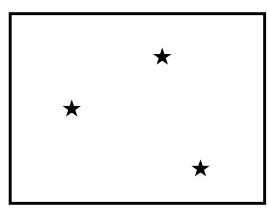

(a)

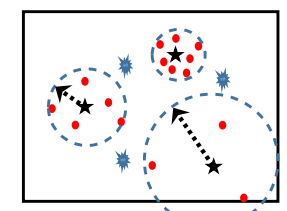

(b) $\cdots \cdot \cdots$

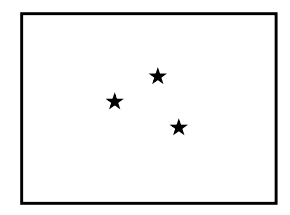

(c)
Fig. 1: The search process of FWA. Black stars are firework individuals, red dots are generated spark individuals and irregularly shaped blue dots are mutant spark individuals. (a) Firework individuals are generated randomly, (b) spark individuals are generated around each firework individual, and mutation sparks are also generated, and (c) new firework individuals are selected from all search individuals in the (b) for the next generation. Steps (b) and (c) are iterated until a termination condition is satisfied.

Because there are several limitations in the original FWA and its performance is also not very prominent among all subsequent variants [13], [14], [15], [16] we use more powerful EFWA as our baseline algorithm and evaluate the performance of our proposed strategy in the following experiments. The enhanced FWA (EFWA) [17] introduces five major improvements into the original FWA to improve its performance without changing the principle and framework of the original FWA.

\section{Proposed Distance-based Exclusive Strategy}

FWA extracts the characteristics of the explosion of real fireworks and simulates these explosion patterns repeatedly to ensure convergence. Firework individuals take on different search capabilities including exploitation and exploration according to their fitness. A better firework individual with higher fitness generates more sparks within a narrow range, while a poor firework individual generates a fewer spark within a wide range. A firework individual and its generated spark individuals locate in a similar area and can be grouped for the same niche area. Inspired by the explosion search, each firework individual would search in a niche area, and no extra operations are required to divide the generated sparks individuals into other niche areas. The core idea of our proposal is to generate individuals into each niche area and make them search independently without overlapping. Finally, each firework individual locates in a different global/local optimum area. Here is the implementation of our proposal in detail.

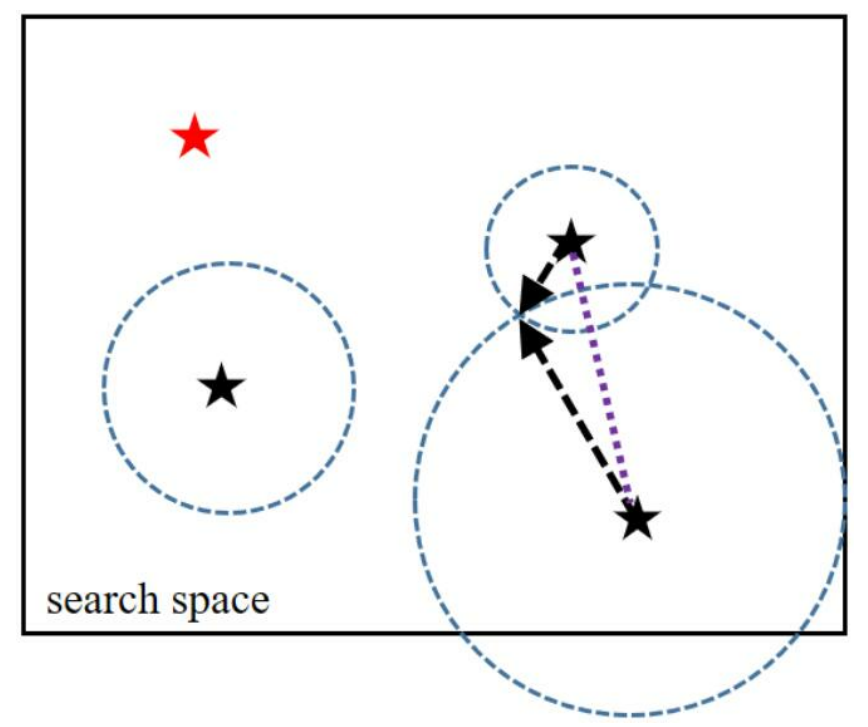

Fig. 2: A demonstration of proposed distance-based exclusive strategy. Once the explosion radius of two firework individuals overlaps, i.e. the sum of black dotted line segments is longer than the purple dotted line segment, the poor firework individual is removed from the local area. Then, a new seaching firework individual (the red five pointed star) is generated to take over an explosion operation of the poor firework individual according to the opposite-based generation strategy.

The key issue is how to avoid overlapping search among firework individuals. To address this issue, we propose a distance-based exclusive strategy to ensure that there is no overlapping search among firework individuals. Since each firework individual has its own explosion radius assigned adaptively according to its fitness, it can be used to detect overlap easily without introducing any new parameters. We determine that there is overlap search between two firework individuals if the Euclidean distance between the two individuals is less than the sum of the search radii of these two individuals.

We check the distances between each firework individual and other fireworks which fitness is better than that of the reference firework and mark the poor firework individual if their searching radius overlaps. Until all firework individuals are sequentially detected, we delete the marked firework individuals and generate new firework individuals to replace them. These generated new firework individuals participate in the next round of explosion operations rather than deleted firework individuals. Note that we use synchronous update detection in this paper. Asynchronous detection is also acceptable and 
may be more reasonable, where a marked firework individual is immediately replaced by a new generated one. In fact, there are many methods to generate new firework individuals. We employ the opposite-based generation strategy shown by the Eq. (1) to generate new firework individuals assigned to unexplored areas as much as possible.

$$
x_{\text {opposite }}^{j}=X_{\max }^{j}+X_{\min }^{j}-x_{i}^{j}
$$

Where $i$ and $j$ mean the $i$-th firework individual and its $j$-th dimension, respectively. $X_{\max }^{j}$ and $X_{\min }^{j}$ are the upper and lower bound of the $j$-th dimension. Thus, $x_{i}^{j}$ and $x_{\text {opposite }}^{j}$ are symmetrical with respect to the center of a search space.

The next point to be solved is how to keep multiple different niche areas as much as possible. Although random selection and distance-based selection are widely used in the original FWA and several its variants, these selection methods may lose the diversity and the ability to search multiple niche areas. To overcome these limitations, local optima-based selection strategy [18] is used to ensure each local optimum firework individual kept in the next generation and maximize the diversity for searching more local optima areas. Note that the mutation operation is not used in our proposal, which means all firework individuals are independent and the optimal individual (spark or current firework) in each niche area is copied to the next generation to form the next population. The Algorithm 1 shows the flow of our proposal combining with FWA.

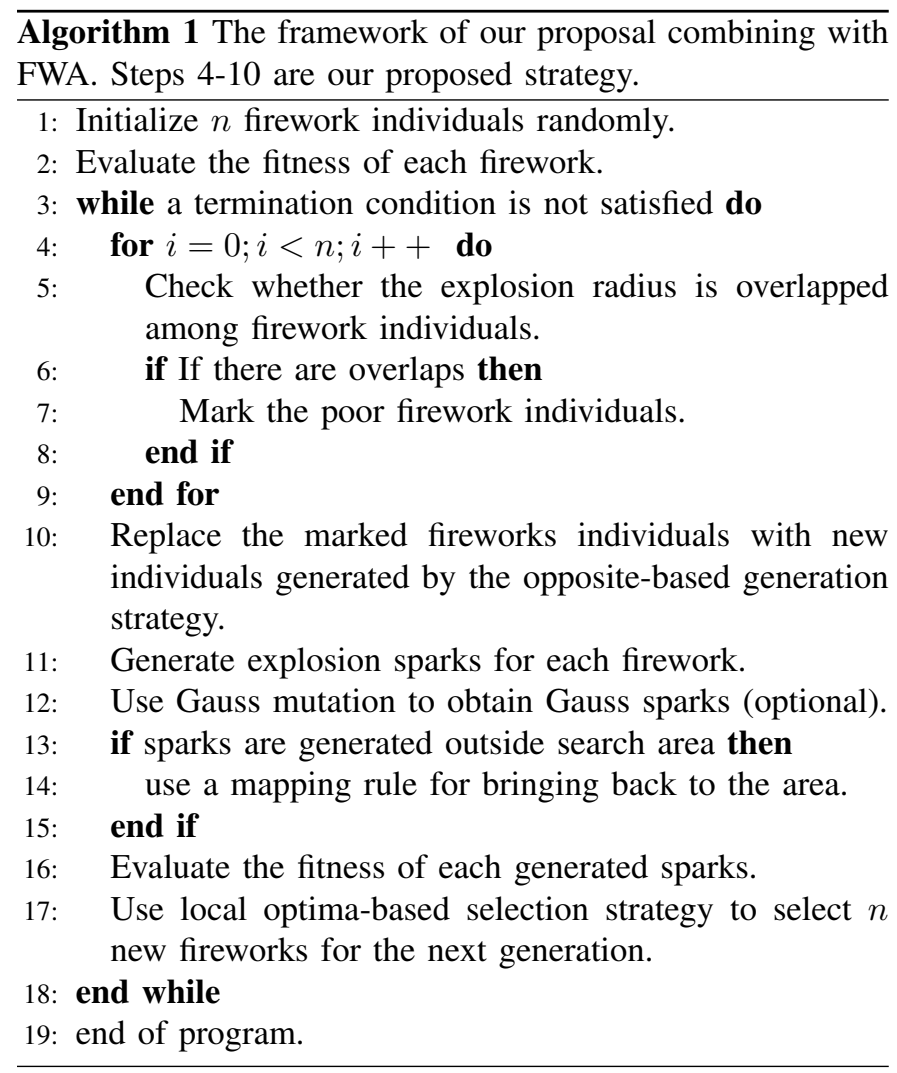

\section{EXPERIMENTAL EVALUATIONS}

We use eight benchmark functions with different dimensions from the CEC2015 multi-niche benchmark test suite [19] in our experimental evaluations; they are designed for real parameter bound constrained single-objective optimization. Table II shows their types, characteristics, variable ranges, and the number of global/local optima. These landscape characteristics include shifted, rotated, combination and multi-modal. The EFWA [17] is used as the test baseline algorithm and combine it with our proposed strategy. The parameter settings used in our experiments are described in the Table I, where the definition of the symbols can be found in the original literatures [3], [17].

TABLE I: Parameter setting of EFWA. D: Dimension

\begin{tabular}{|l|c|}
\hline \multicolumn{1}{|c|}{ Parameters } & Values \\
\hline \# of fireworks for any dimension search & 10 \\
\hline \# of sparks $m$ & 50 \\
\hline \# of Gauss mutation sparks & 5 \\
\hline constant parameters & $a=0.04 b=0.8$ \\
\hline Maximum amplitude $A_{\max }$ & 20 \\
\hline Dimensions $D$ & $2,3,4,5,6,8,10,16$ and 20 \\
\hline Max. \# of fitness evaluations & $20,000 D$ \\
\hline \# of trial runs & 30 \\
\hline
\end{tabular}

TABLE II: Benchmark Function.

\begin{tabular}{|c|c|c|c|}
\hline No. & Functions & Dimension & \# of global/local \\
\hline \multirow{3}{*}{$F_{1}$} & Shifted and Rotated Expanded & 5 & $1 / 15$ \\
& Two-Peak Trap & 10 & $1 / 55$ \\
& & 20 & $1 / 210$ \\
\hline \multirow{3}{*}{$F_{2}$} & Shifted and Rotated Expanded & 2 & $4 / 21$ \\
& Five-Uneven-Peak Trap & 5 & $32 / 0$ \\
& & 8 & $256 / 0$ \\
\hline \multirow{3}{*}{$F_{3}$} & Shifted and Rotated Expanded & 2 & $25 / 0$ \\
& Equal Minima & 3 & $125 / 0$ \\
& & 4 & $625 / 0$ \\
\hline \multirow{3}{*}{$F_{4}$} & Shifted and Rotated Expanded & 5 & $1 / 15$ \\
& Decreasing Minima & 10 & $1 / 55$ \\
& & 20 & $1 / 210$ \\
\hline \multirow{2}{*}{$F_{5}$} & Shifted and Rotated Expanded & 2 & $25 / 0$ \\
& Uneven Minima & 3 & $125 / 0$ \\
& & 4 & $625 / 0$ \\
\hline \multirow{2}{*}{$F_{6}$} & Shifted and Rotated Expanded & 4 & $16 / 0$ \\
& Himmelblau's Function & 6 & $64 / 0$ \\
& & 8 & $256 / 0$ \\
\hline \multirow{2}{*}{$F_{7}$} & Shifted and Rotated Expanded & 6 & $8 / 0$ \\
& Six-Hump Camel Back & 10 & $32 / 0$ \\
& & 16 & $256 / 0$ \\
\hline \multirow{2}{*}{$F_{8}$} & Shifted and Rotated Modified & 2 & $36 / 0$ \\
& Vincent Function & 3 & $216 / 0$ \\
& & 4 & $1296 / 0$ \\
\hline \multirow{2}{*}{} & & & \\
\hline
\end{tabular}

To evaluate the performance of our proposal, we not only compare it with the conventional EFWA, but also with a wellknown niching method, fitness sharing [20], [21]. The basic idea of fitness sharing is to restrict and share resources (e.g. fitness value) at each niche for decreasing redundancy in the population. Since the search range in all dimensions is limited to $[-100,100]$, we set the niche radius at a fixed value 5 for fitness sharing in our experiments. It means that if a distance 
between two firework individuals is less than the niche radius, it is regarded that they are in the same area and thus need to share fitness. Fitness sharing is used to reassign fitness for firework individuals before the explosion operation is performed. Then, the reassigned fitness of firework individuals is used to determine the number of generated sparks and their explosion radius. The above description demonstrates how to integrate fitness sharing into the conventional EFWA.

We evaluate convergence against the number of fitness calls rather than generations for fair evaluations. We test each benchmark function with 30 trial runs in different dimensional spaces. The fitness accuracy error is set to $10^{-4}$, which means that if the error between a found optimum and a true optimum is less than this value, then the found optimum is considered to converge to the true optimum. The maximum number of fitness calculations is set to $20,000 D$, where $D$ is the spatial dimension.

To analyze the performance among these algorithms, we record the average number of found optima after the 30 trial runs, as well as the maximum and minimum number of found optima at the stop condition, e.g. the maximum number of fitness calculations. The results are shown in the Table III. We apply Friedman test and Holm's multiple comparison for average number of found optima at the stop condition of three methods to check the significance difference among them. The Tables IV shows the statistical test result of average number of the found optima.

\section{Discussions}

We begin our discussion on an explanation of the superiority of our proposed strategy. Our proposal uses the explosion pattern of FWA fully to make each firework individual stay in a different niche area. The independent selection mechanism can keep the diversity of the population and ensure that multiple niche areas are kept well.

In comparison to other existing niching methods, the proposed strategy does not introduce any new parameters to divide individuals into different niche areas because each local explosion operation can be considered a niche. The proposed strategy needs extra fitness calculations to generate new firework individuals when search areas are overlapped. However, it can avoid multiple firework individuals searching in the same local area repeatedly, and the possibility that new generated firework individuals locate in an unexplored area becomes high. Besides, our proposal always keeps the optimal area unreplaced, and the poorer the area is, the higher the possibility that the area is cleared is. Thus, this strategy can avoid the wandering search in the same area and keep multiple niches with low increased cost. We can say that it is a low risk, high return strategy.

Secondly, we want to discuss on the potential and usability of our proposal. The proposed strategy can be combined with any other FWA variations easily to track multiple different niches without changing their main framework; it is not limited to EFWA used in our experiments. It can be further extended to other EC algorithms. For example, each particle in PSO has a maximum evolutionary step (maximum speed) that can be used to detect whether the particles located in the same local area. Subsequently, worse particles in the same local area are replaced with newly generated particles to keep multiple niche search. It is only a preliminary idea, and a more perfect framework still needs to be discussed. The opposite-based generation strategy is adopted to generate new firework individuals in this paper, but other generation strategies are also acceptable, e.g. random generation, Forex trade strategy generation, and others. Thus, how to generate firework individuals appropriately at different search stages can be developed as a new potential topic. Overall, we can say that the proposed strategy has a strong plasticity and promising.

To analyze the performance of our proposal, the Friedman test and Holm's multiple comparison are applied to check the significant differenceat the stop condition. From the results of the statistical tests, we have found that our proposal does not weaken the performance of FWA on all benchmark functions, and satisfactory performance was obtained on $F_{2}, F_{3}, F_{5}, F_{6}$ and $F_{8}$. On the other hand, fitness sharing does not play any role in finding multiple optima. It may be because it can reallocate resources, i.e. explosion radius of a firework and the number of its generated sparks, to avoid duplicate searches, but it cannot keep multiple niche search with convergence and converge to the same local optimum area. It also implies that our proposal can keep diversity well. Both, $F_{1}$ and $F_{4}$ have only one global optimum and multiple local optima. None of three algorithms converge to the global optimal area of these two benchmark functions, which may be caused by the fact that the global optimum is hard to find. Our proposal had no effect on $F_{7}$. Its specific reasons are still unclear, and we will investigate in our future work, which may help us to design more reasonable strategies to optimize multimodal problems.

Some open topics and possible improvements are given. Thanks to the explosion search of FWA, it can be developed easily for multimodal optimization problems. But, it limits the population size not to be set too large because abundant resources (fitness evaluations) are used to generate spark individuals for local search. The local optima-based selection strategy may facilitate the implementation of parallel computing, though it is not conducive to exchange information among firework individuals and not easy to jump out of local optima. Things have pro and con, and it is necessary to further consider how to balance these conflicts to pursue maximum performance. Thus, our proposal can ensure each firework individual may locate an optimum, that determines that it is not suitable for optimization problems with massive optima.

Finally, we want to discuss on parameter settings. Since there are no mature methods to guide the parameter settings, all parameters used in experiments are set based on our experiences. Actually, adaptive detection mechanisms are potential approaches rather than a fixed strategy according to the convergence progresses or the characteristics of a fitness landscape. Thus, developing an adaptive version has become a challenge in our future works. 
TABLE III: We record the mean of 30 trial runs, as well as the maximum and minimum number of found optima at the stop condition. EFWA: enhanced FWA; EFWAWithFS: apply fitness sharing to EFWA; Poposal: apply our proposed strategy to EFWA.

\begin{tabular}{|c|c|c|c|c|c|c|c|c|c|c|}
\hline \multirow{2}{*}{ No. } & \multirow{2}{*}{ Dimension } & \multicolumn{3}{|c|}{ EFWA } & \multicolumn{3}{|c|}{ EFWAWithFS } & \multicolumn{3}{|c|}{ Proposal } \\
\hline & & Mean & Min. & Max. & Mean & Min. & Max. & Mean & Min. & Max. \\
\hline \multirow{3}{*}{$\mathrm{F} 1$} & 5 & 0 & 0 & 0 & 0.033333 & 0 & 1 & 0 & 0 & 0 \\
\hline & 10 & 0 & 0 & 0 & 0 & 0 & 0 & 0 & 0 & 0 \\
\hline & 20 & 0 & 0 & 0 & 0 & 0 & 0 & 0 & 0 & 0 \\
\hline \multirow{3}{*}{$\mathrm{F} 2$} & 2 & 1 & 1 & 1 & 1 & 1 & 1 & 2.166667 & 1 & 4 \\
\hline & 5 & 0.833333 & 0 & 1 & 0.933333 & 0 & 1 & 4.933333 & 2 & 9 \\
\hline & 8 & 0.3 & 0 & 1 & 0.366667 & 0 & 1 & 1.933333 & 0 & 4 \\
\hline \multirow{3}{*}{ F3 } & 2 & 1 & 1 & 1 & 1 & 1 & 1 & 7.133333 & 4 & 9 \\
\hline & 3 & 1 & 1 & 1 & 1 & 1 & 1 & 3.333333 & 1 & 7 \\
\hline & 4 & 1 & 1 & 1 & 0.9 & 0 & 1 & 1.4 & 0 & 5 \\
\hline \multirow{3}{*}{$\mathrm{F} 4$} & 5 & 0.033333 & 0 & 1 & 0 & 0 & 0 & 0 & 0 & 0 \\
\hline & 10 & 0 & 0 & 0 & 0 & 0 & 0 & 0 & 0 & 0 \\
\hline & 20 & 0 & 0 & 0 & 0 & 0 & 0 & 0 & 0 & 0 \\
\hline \multirow{3}{*}{ F5 } & 2 & 1 & 1 & 1 & 1 & 1 & 1 & 6.833333 & 4 & 9 \\
\hline & 3 & 1 & 1 & 1 & 1 & 1 & 1 & 5.966667 & 3 & 8 \\
\hline & 4 & 1 & 1 & 1 & 0.933333 & 0 & 1 & 1.833333 & 0 & 6 \\
\hline \multirow{3}{*}{ F6 } & 4 & 1 & 1 & 1 & 1 & 1 & 1 & 8.033333 & 6 & 9 \\
\hline & 6 & 1 & 1 & 1 & 1 & 1 & 1 & 9 & 9 & 9 \\
\hline & 8 & 1 & 1 & 1 & 1 & 1 & 1 & 9 & 9 & 9 \\
\hline \multirow{3}{*}{ F7 } & 6 & 0.266667 & 0 & 1 & 0.233333 & 0 & 1 & 0.233333 & 0 & 1 \\
\hline & 10 & 0.033333 & 0 & 1 & 0.033333 & 0 & 1 & 0 & 0 & 0 \\
\hline & 16 & 0 & 0 & 0 & 0 & 0 & 0 & 0 & 0 & 0 \\
\hline \multirow{3}{*}{ F8 } & 2 & 1.8 & 1 & 6 & 1.833333 & 1 & 7 & 6.4 & 3 & 9 \\
\hline & 3 & 1.633333 & 1 & 5 & 1.333333 & 1 & 3 & 8.766667 & 8 & 9 \\
\hline & 4 & 1.4 & 1 & 4 & 1.133333 & 1 & 3 & 8.4 & 6 & 9 \\
\hline
\end{tabular}

\section{CONCLUSION}

We introduced a distance-based exclusive strategy to FWA to solve multimodal tasks. Each individual can stay in different niche area and can converge to different optima. The experiments have confirmed that our proposed strategy is effective and can ensure that FWA locates in multiple different optima areas.

In our future work, we will consider the fitness characteristics of optimization problems and use the collected feedback in EC process to develop a more intelligent version to improve performance of our proposal.

\section{ACKNOWLEDGMENT}

This work was supported in part by Grant-in-Aid for Scientific Research (18K11470, 17H06197) and the Natural Science Foundation of China (NSFC) under grant no. 61673025.

\section{REFERENCES}

[1] R. Storn and K. Price, "Differential evolution-a simple and efficient heuristic for global optimization over continuous spaces," Journal of global optimization, vol. 11, no. 4, pp. 341-359, 1997.

[2] J. Kennedy and R. Eberhart, "Particle swarm optimization," in IEEE International Conference on Neural Networks - Conference Proceedings, vol. 4, Perth, WA, Australia, December 1995, pp. 1942-1948.
[3] Y. Tan and Y. Zhu, "Fireworks algorithm for optimization," ser. Lecture Notes in Computer Science, vol. 6145 LNCS, no. PART 1, Beijing, China, June 2010, pp. 355-364.

[4] X. Yang and D. Suash, "Cuckoo search via lévy flights," in 2009 World Congress on Nature and Biologically Inspired Computing, NABIC 2009 - Proceedings, 2009, pp. 210-214.

[5] X. Yang and A. H. Gandomi, "Bat algorithm: A novel approach for global engineering optimization," Engineering Computations, vol. 29, no. 5, pp. 464-483, 2012.

[6] A. H. Gandomi and A. H. Alavi, "Krill herd: A new bio-inspired optimization algorithm," Communications in Nonlinear Science and Numerical Simulation, vol. 17, no. 12, pp. 4831-4845, 2012.

[7] A. Petrowski, "A clearing procedure as a niching method for genetic algorithms," in Proceedings of IEEE International Conference on Evolutionary Computation, Nagoya, Japan, May 1996, pp. 798-803.

[8] G. Harik, "Finding multimodal solutions using restricted tournament selection," in Proceedings of the 6th International Conference on Genetic Algorithms, San Francisco, CA, USA, July 1995, pp. 24-31.

[9] K. A. De Jong, "An analysis of the behavior of a class of genetic adaptive systems," in PhD thesis, University of Michigan, Ann Arbor, MI, USA, 1975.

[10] E. G. David and R. Jon, "Genetic algorithms with sharing for multimodal function optimization," in Proceedings of the Second International Conference on Genetic algorithms and their application, Hillsdale, NJ, USA, July 1987, pp. 41-49.

[11] J. Li, M. E. Balazs, G. T. Parks, and P. J. Clarkson, "A species conserving genetic algorithm for multimodal function optimization," Evolutionary Computation, vol. 10, no. 3, pp. 207-234, 2002.

[12] M. Bessaou, A. Petrowski, and P. Siarry, "Island model cooperating with speciation for multimodal optimization," in 6th International Conference 
TABLE IV: Statistical test results of the Friedman test and Holm's multiple comparison for average number of found optima among three algorithms at the stop condition. $A \gg B$ and $A>B$ mean that $A$ is significantly better than $B$ with significant levels of $1 \%$ and $5 \%$, respectively. $A \approx B$ means that there is no significant difference between them although $A$ is better than $B$. The abbreviation used in this table is the same with those used in the Table III.

\begin{tabular}{|c|c|c|}
\hline No. & Dimension & Statistical test results \\
\hline \multirow{3}{*}{ F1 } & 5 & EFWAWithFS $\approx$ Proposal $\approx$ EFWA \\
\hline & 10 & EFWAWithFS $\approx$ Proposal $\approx$ EFWA \\
\hline & 20 & EFWAWithFS $\approx$ Proposal $\approx$ EFWA \\
\hline \multirow{3}{*}{$\mathrm{F} 2$} & 2 & Proposal $\gg$ EFWAWithFS $\approx$ EFWA \\
\hline & 5 & Proposal $\gg$ EFWAWithFS $\approx$ EFWA \\
\hline & 8 & Proposal $\gg$ EFWAWithFS $\approx$ EFWA \\
\hline \multirow{3}{*}{ F3 } & 2 & Proposal $\gg$ EFWAWithFS $\approx$ EFWA \\
\hline & 3 & Proposal $\gg$ EFWAWithFS $\approx$ EFWA \\
\hline & 4 & Proposal $\approx$ EFWA $\approx$ EFWAWithFS \\
\hline \multirow{3}{*}{$\mathrm{F} 4$} & 5 & EFWA $\approx$ Proposal $\approx$ EFWAWithFS \\
\hline & 10 & EFWA $\approx$ Proposal $\approx$ EFWAWithFS \\
\hline & 20 & EFWA $\approx$ Proposal $\approx$ EFWAWithFS \\
\hline \multirow{3}{*}{ F5 } & 2 & Proposal $\gg$ EFWA $\approx$ EFWAWithFS \\
\hline & 3 & Proposal $\gg$ EFWA $\approx$ EFWAWithFS \\
\hline & 4 & Proposal $\gg$ EFWA $\approx$ EFWAWithFS \\
\hline \multirow{3}{*}{ F6 } & 4 & Proposal $\gg$ EFWA $\approx$ EFWAWithFS \\
\hline & 6 & Proposal $\gg$ EFWA $\approx$ EFWAWithFS \\
\hline & 8 & Proposal $\gg$ EFWA $\approx$ EFWAWithFS \\
\hline \multirow{3}{*}{ F7 } & 6 & EFWA $\approx$ EFWAWithFS $\approx$ Proposal \\
\hline & 10 & EFWA $\approx$ EFWAWithFS $\approx$ Proposal \\
\hline & 16 & EFWA $\approx$ EFWAWithFS $\approx$ Proposal \\
\hline \multirow{3}{*}{ F8 } & 2 & Proposal $\gg$ EFWAWithFS $\approx$ EFWA \\
\hline & 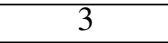 & Proposal $\gg$ EFWA $\approx$ EFWAWithFS \\
\hline & 4 & Proposal $\gg$ EFWA $\approx$ EFWAWithFS \\
\hline
\end{tabular}

on Parallel Problem Solving from Nature, 2000, pp. 437-446.

[13] J. Yu, Y. Tan, and H. Takagi, "Accelerating fireworks algorithm with an estimated convergence point," in Proceedings of 9th International Conference on Swarm Intelligence, Shanghai, China, 2018, pp. 263484.

[14] J. Yu and H. Takagi, "Acceleration for fireworks algorithm based on amplitude reduction strategy and local optima-based selection strategy," in Proceedings of 8th International Conference on Swarm Intelligence, Fukuoka, Japan, 2017, pp. 477-484.

[15] J. Yu, Y. Tan, and H. Takagi, "Scouting strategy for biasing fireworks algorithm search to promising directions," in GECCO 2018 Companion - Proceedings of the 2018 Genetic and Evolutionary Computation Conference Companion, 2018, pp. 99-100.

[16] J. Yu, H. Takagi, and Y. Tan, "Multi-layer explosion-based fireworks algorithm," International Journal of Swarm Intelligence and Evolutionary Computation, vol. 7, no. 3, 2018. [Online]. Available: DOI: $10.4172 / 2090-4908.1000173$

[17] S. Zheng, A. Janecek, and Y. Tan, "Enhanced fireworks algorithm," in 2013 IEEE Congress on Evolutionary Computation, CEC 2013, Cancun, Mexico, June 2013, pp. 2069-2077.

[18] J. Yu and H. Takagi, "Acceleration for fireworks algorithm based on amplitude reduction strategy and local optima-based selection strategy," ser. Lecture Notes in Computer Science (including subseries Lecture Notes in Artificial Intelligence and Lecture Notes in Bioinformatics), vol. 10385 LNCS, Fukuoka, Japan, July 2017, pp. 477-484.

[19] B. Y. Qu, J. J. Liang, P. N. Suganthan, and Q. Chen, "Problem definitions and evaluation criteria for the cec 2015 competition on single objective multi-niche optimization," 2015. [Online]. Available: http://web.mysites.ntu.edu.sg/epnsugan/PublicSite/Shared\%
20Documents/CEC-2015/Niching-Benchmark/Definitions\%20of\%20\% 20CEC2015\%20niching\%20benchmark\%2020141228.pdf

[20] D. E. Goldberg and J. Richardson, "Genetic algorithms with sharing for multimodal function optimization," in Proceedings of the Second International Conference on Genetic Algorithms on Genetic algorithms and their application, Cambridge, USA, July 1987, pp. 41-49.

[21] B. Sareni and L. Krhenbhl, "Fitness sharing and niching methods revisited," IEEE Transactions on Evolutionary Computation, vol. 2, no. 3, pp. 97-106, 1998. 